\title{
Et si l'Afrique refusait le développement?, Axelle Kabou, l'Harmattan 1991,208 p.
}

\section{Philippe Lavigne Delville}

\section{Q OpenEdition}

\section{Journals}

Édition électronique

URL : http://journals.openedition.org/apad/416

DOI : 10.4000/apad.416

ISSN : 1950-6929

Éditeur

LIT Verlag

Édition imprimée

Date de publication : 15 décembre 1991

\section{Référence électronique}

Philippe Lavigne Delville, «Et si l'Afrique refusait le développement?, Axelle Kabou, l'Harmattan 1991,208 p. », Bulletin de l'APAD [En ligne], 2 I 1991, mis en ligne le 06 juillet 2006, consulté le 21 septembre 2020. URL : http://journals.openedition.org/apad/416 ; DOI : https://doi.org/10.4000/apad.416

Ce document a été généré automatiquement le 21 septembre 2020.

Bulletin de l'APAD 


\title{
Et si l'Afrique refusait le développement?, Axelle Kabou, l'Harmattan 1991,208 p.
}

\author{
Philippe Lavigne Delville
}

1 "Et si l'Afrique refusait le développement ?". Le sous-développement n'est pas dû un manque de capitaux, aucune des interprétations économiques ne parvient à rendre compte de la situation. Dès lors, il faut bien revenir à la question taboue des "mentalités". Mais attention, pas des mentalités africaines à la Griaule : "le refus du développement fleurit moins sur le terrain de la tradition villageoise que sur la macadam des capitales". "Ces comportements et attitudes suicidaires trop hâtivement assimilés à des persistances de cultures traditionnelles constituent un nouveau système idéologique implicitement revendiqué par une élite africaine honteuse de son "occidentalité"" , qui a besoin de "se laver du péché de toubabisation". C'est bien des mentalités actuelles des classes dominantes que parle Axelle Kabou.

2 L'Afrique n'a pas su dépasser son "sanglot de l'homme noir". Elle s'est construite une image d'elle-même en éternelle victime, où la traite, la colonisation, puis les termes de l'échange sont les seules causes des difficultés. Les théories de la négritude, loin de réhabiliter l'homme noir, l'a enfermé dans un "droit à la différence" qui n'était qu'un droit à l'auto-marginalisation. "L'Afrique moderne paraît avoir autant de mal à revendiquer une modernité associée à la traite négrière et à la colonisation qu'à assumer la totalité d'un système anté-colonial à qui elle reproche deux défaites retentissantes". "Les Africains restent largement persuadés que leur destin doit être pris en charge par des étrangers", que "les prétentions civilisatrices de l'Occident ne s'arrêtent pas avec les indépendances". Dès lors, "l'Afrique n'est pas loin de ressentir le développement comme une injonction", "les efforts de développement sont perçus comme des aveux d'impuissance, d'infériorité culturelle ou raciale". "L'Afrique noire reste profondément humiliée par l'idée même de développement", considérant que c'est "une tâche qui relève légitimement des obligations du colonisateur". La technique 
reste perçue comme "la chose du blanc", qu'on utilise plus ou moins dédaigneusement, sans chercher à s'en emparer.

3 Le système du "développement", dimension structurelle de l'économie post-indépendantiste, n'a fait que renforcer ces tendances: "Trente années de télé-alimentation, de télé-financement, de détournements divers ont, semble-t-il, largement convaincu les bourgeois africains que leur argent ne saurait servir au développement de leur continent: c'est-là le rôle historiquement dévolu à l'ancien colonisateur". Pourtant, "les Africains qui ressentent leur retard comme une accusation implicite d'arriération par rapport à l'Occident et qui se barricadent derrière leurs valeurs culturelles doivent savoir qu'ils se tendent là un piège dont ils sont les seuls à pâtir".

4 Pour Axelle Kabou, ce refus du développement découle directement du système idéologique post-indépendances, basé sur ce qu'elle appelle le "vendredisme" : dans le roman de Defoe, Vendredi symbolise à la fois le bon sauvage et le complexe de dépendance $d u$ primitif à l'égard de l'homme blanc. Les "mythes post-indépendantistes" se sont donc constitués dans la période charnière des indépendances. Le complexe de la colonisation a conduit les élites africaines à jeter le bébé de l'emprunt technologique avec le bain de l'impérialisme. Les critiques internes à l'Occident, tiers-mondisme et critique marxiste en particulier, rebondissant sur les théories de la négritude, leur ont fourni le prêt-à-penser idéologique permettant à la fois de rejeter l'ensemble des responsabilités sur l'Occident, de mettre en place le système de dépendance économique et de se survaloriser culturellement. Le relativisme culturel achève l'inversion symbolique du rapport de dépendance. Il permet $d$ 'invoquer un droit à la différence qui "se manifeste par une sorte de détermination altière à n'être que soi et rien d'autre, et surtout à n'y voir aucun inconvénient, quand bien même le plus grand exploit en matière d'auto-réhabilitation ne consisterait qu'à diaboliser les idées nouvelles, à ériger la mendicité en principe de développement et à liquider les gêneurs".

5 L'Occident a lui aussi sa part de responsabilité. Le vendredisme des élites africaines a rencontré un soutien, explicite ou implicite de la part des pays occidentaux, à travers le sanglot de l'homme blanc ou la logique internationale qui a institutionnalisé le "développement". Sans même parler de l'appui politique à des régimes douteux sous prétexte de guerre froide, "les spécialistes du développement, dévorés par leurs propres passions, aveuglés par les refrains à la mode, ont pratiqué, à gauche comme à droite, la flagornerie, le paternalisme ou opté pour la fuite tangentielle quand il s'agissait des vrais débats du sous-développement de l'Afrique" •

6 Mais le sanglot de l'homme blanc est passé. "L'Afrique a-t-elle retenu que [le tiers-mondisme] répond essentiellement à un besoin de liquider les contentieux issus de la décolonisation et de la guerre froide, pour permettre à une Europe (enfin réconciliée avec la civilisation technicienne) d'aborder le XXI ${ }^{\mathrm{e}}$ siècle avec des habits neufs? Tout porte à croire que non. En retard de trois longueurs, comme d'habitude, l'Afrique ne semble pas comprendre les implications profondes de la bourrasque qui secoue l'Occident en ce moment, et s'obstine à lancer des appels à l'aide en direction de l'Europe occidentale, non seulement déterminée à tiers-mondiser une Europe centrale dégoûtée du socialisme soviétique, mais aussi décidée à considérer désormais l'aide au développement comme un simple geste de charité". La question est clairement posée : "l'Afrique est enfin condamnée à se débrouiller. Doit-on vraiment le déplorer ?" 
7 La crise actuelle montre que "les mythes post-coloniaux créés par la génération des indépendances ont désormais atteint leur rendement maximum en tant que facteurs de régulation sociale". "La liste des ruptures socio-économiques actuellement perceptibles en Afrique est longue (...) l'effondrement des supports économiques habituels d'une société essentiellement combinarde, désormais sciés à la base, installe partout une angoisse polymorphe, pesante, lourde de menaces pour l'avenir immédiat. (...) Les sources "modernes" d'alimentation et de régénération des solidarités atomisées sont à peu près épuisées". "La décennie 1990-2000 sera sanglante en Afrique". "Il est difficile de croire que de tels revirement restent sans effet sur les mentalités. (...) Le tout est de savoir combien de temps il faudra pour qu'éclate à l'échelle du continent la révolution sociale salutaire".

8 "Face à un Occident débarrassé de sa mauvaise conscience à l'égard des sous-développés, l'Afrique a peut-être une chance de comprendre que le droit à n'être que soi est la rançon d'un long et patient effort de vitalisation et de revitalisation du patrimoine culturel par l'intégration intelligente d'éléments nouveaux, étrangers ou non". Axelle Kabou prône une Afrique qui s'assume elle-même, pratique largement et sans complexe les emprunts à l'Occident avec un "opportunisme scientifique" semblable à celui du Japon, qui arrive enfin à dépasser "l'absence cruelle de projet de société cohérent".

Par son ton et son discours, Axelle Kabou, jeune camerounaise de 36 ans, se veut le porte-parole "d'une génération objectivement privée d'avenir, qui a tout intérêt à travailler à l'effondrement des nationalismes étroits des indépendances et à l'avenir d'une Afrique large, forte et digne". C'est bien comme cela qu'il faut lire ce livre. Il enfonce des tabous et, en ce sens, il est salutaire. Mais il ne faudrait pas qu'une lecture trop rapide, enchantée par le style alerte et le sens de la formule, croient retrouver-là l'afro-pessismisme bon teint des salons parisiens. D'abord parce qu'il ne renie pas la puissance d'analyse des théories de la dépendance, cite Samir Amin et Franz Fanon, regrettant même qu'ils aient été tellement vidés de leur substance. Ensuite parce que, en tant que critique politique interne, il représente l'émergence d'une nouvelle classe politique qui veut achever un système postindépendances pervers et moribond. Il y a là quelque chose de sain que n'a pas le discours ultra-libéral occidental, même si, une fois de plus, les discours propres à l'Occident peuvent servir dans les enjeux locaux africains. 